Pacific Journal of Mathematics

ON THE REGULARITY UP TO THE BOUNDARY FOR SECOND 


\title{
ON THE REGULARITY UP TO THE BOUNDARY FOR SECOND ORDER NONLINEAR ELLIPTIC SYSTEMS
}

\author{
M. Giaquinta, J. NeČas, O. John, and J. Sttará
}

It is proved the regularity up to the boundary of the uniformly Lipschitz-continuous weak solutions of a boundary value problem for the elliptic system

$$
-D_{i} a_{i}^{r}(x, u, D u)+\bar{a}^{r}(x, u, D u)=f^{r} ; \quad r=1, \cdots, m
$$

from the Liouville properties of the system.

In (1.1) $u=\left\{u^{r}\right\}_{r=1, \cdots, m}$ is a vector function and $D u=\left\{D_{i} u^{r}\right\}_{\substack{i=1, \ldots, n \\ r=1, \cdots, m}}$ is its gradient. We write $D_{i} u^{r}=\partial u^{r} / \partial x_{i}$ and the summation convention is used throughout the paper.). We follow the ideas of our previous work (see [1-4]) where interior regularity was shown to be equivalent (in some sense) to the Liouville property $(L)$ (see Definition 2.2). In the present paper, regularity up to the boundary is shown to be, essentially, equivalent to the previous $(L)$ together with a certain "boundary" Liouville property $\left(L^{+}\right)$(see Definition 2.3).

2. Notation and assumptions. Let $\boldsymbol{R}^{n}$ be an $n$-dimensional Euclidean space; for $x=\left(x_{1}, \cdots, x_{n-1}, x_{n}\right)=\left(x^{\prime}, x_{n}\right) \in \boldsymbol{R}^{n}$ let $|x|=$ $\max \left\{\left|x_{i}\right| ; i=1, \cdots, n\right\} ;$ further let $\boldsymbol{R}_{+}^{n}=\left\{x \in \boldsymbol{R}^{n} ; x_{n}>0\right\} ; \Omega=\left\{x \in \boldsymbol{R}_{+}^{n}\right.$; $|x|<1\} ; \Gamma=\left\{x \in \boldsymbol{R}^{n} ;\left|x^{\prime}\right|<1 ; x_{n}=0\right\} ; B\left(x_{0}, R\right)=\left\{x \in \Omega ;\left|x-x_{0}\right|<R\right\} ;$ $\Gamma\left(x_{0}, R\right)=\overline{B\left(x_{0}, R\right)} \cap \Gamma$.

Let us denote

$$
\begin{aligned}
a(x, u, D u) & =\left\{a_{i}^{r}(x, u, D u)\right\}_{\substack{i=1, \cdots, n \\
r=1, \cdots, m}} \\
\bar{a}(x, u, D u) & =\left\{\bar{a}^{r}(x, u, D u)\right\}_{r=1, \cdots, m} \\
f(x) & =\left\{f^{r}(x)\right\}_{r=1, \cdots, m},
\end{aligned}
$$

where $a, \bar{a}$ are once continuously differentiable functions on $\bar{\Omega} \times$ $\boldsymbol{R}^{m} \times \boldsymbol{R}^{n m}$, and $f \in\left[W^{1, p / 2}(\Omega)\right]^{m}$ for some $p, p>n$.

REMARK. In what follows we omit the notation of the Cartesian product. So we write $f \in W^{1, p}(\Omega)$ instead of $f \in\left[W^{1, p}(\Omega)\right]^{m n}$ etc.

In this notation the system (1.1) can be rewritten as

$$
-\operatorname{div}(a(x, u, D u))+\bar{a}(x, u, D u)=f(x)
$$

on $\Omega$. We suppose that the strong ellipticity condition holds:

$$
\frac{\partial a_{i}^{r}}{\partial \eta_{j}^{s}}(x, \xi, \eta) \zeta_{i}^{r} \zeta_{j}^{s}>0
$$


for every $\zeta \neq 0$ and each $(x, \xi, \eta) \in \bar{\Omega} \times \boldsymbol{R}^{m} \times \boldsymbol{R}^{n m}$.

To describe the boundary conditions we introduce two disjoint sets $M, N$ of positive integers such that $M \cup N=\{1, \cdots, m\}$ (both the cases $M=\varnothing$ and $N=\varnothing$ being admissible). Let $\left\{b_{r s}\right\}_{r \in M, s \in N}$ be the set of real constants. The stable boundary operators $B_{r}(r \in M)$ are given by the formulas

$$
B_{r} u=u^{r}-\sum_{s \in N} b_{r s} u_{s} .
$$

Put

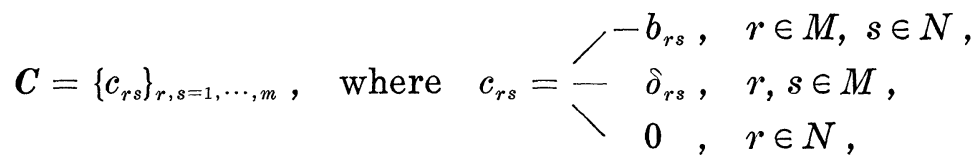

$$
\begin{aligned}
& C^{*}=\left\{c_{r s}^{*}\right\}_{r, s=1, \cdots, m}, \quad \text { where } \quad c_{r s}^{*}=\begin{array}{ll}
b_{s r}, & r \in N, s \in M \\
\delta_{r s}, & r, s \in N, \\
0 & r \in M,
\end{array} \\
& \mathscr{F}(x, u, D u)=\left\{a_{n}^{r}(x, u, D u)+h^{r}(x, u)-g^{r}(x)-f_{n}^{r}(x)\right\}_{r=1, \cdots, m},
\end{aligned}
$$

where $h$ and $g$ are given functions; $h \in C^{1}\left(\bar{\Gamma} \times \boldsymbol{R}^{m}\right), g \in W^{1, \infty}(\Gamma)$.

Let, finally, $u_{0}=\left\{u_{0}^{r}\right\}_{r=1, \cdots, m}$ be a given function from $W^{2, p}(\Omega)$. We consider the following boundary value problem for the system (2.1) (in its classical formulation):

$$
\begin{aligned}
& C\left(u-u_{0}\right)=0 \text { on } \Gamma, \\
& C^{*} \mathscr{F}(x, u, D u)=0 \text { on } \Gamma, \\
& u-u_{0}=0 \text { on } \partial \Omega \backslash \Gamma .
\end{aligned}
$$

Denote the scalar product in $\boldsymbol{R}^{n}$ as well as in $\boldsymbol{R}^{n m}$ by $($,$) and put$

$$
V=\left\{v \in W^{1,2}(\Omega) ; \quad \boldsymbol{C} v=0 \quad \text { on } \quad \Gamma ; \quad v=0 \quad \text { on } \quad \partial \Omega \backslash \Gamma\right\} .
$$

A function $u \in W^{1,2}(\Omega)$ is said to be a weak solution of the problem (2.1), (2.4) if

$$
\begin{gathered}
\text { (i) } u-u_{0} \in V, \\
\text { (ii) for each } \varphi \in V \text {, it holds } \\
\int_{\Omega}\{(a, D \varphi)+(\bar{\alpha}, \varphi)-(f, \varphi)\} d x=\int_{\Gamma}(h-g, \varphi) d x^{\prime} .
\end{gathered}
$$

(Let us rewrite for once the equation (2.6) (ii) in a more detailed form:

(2.6) (ii) ${ }^{\prime}$

$$
\begin{gathered}
\int_{\Omega}\left\{a_{i}^{r}(x, u(x), D u(x)) D_{i} \varphi^{r}(x)+\bar{a}^{r}(x, u(x), D u(x)) \varphi^{r}(x)-f^{r}(x) \varphi^{r}(x)\right\} d x \\
=\int_{\Gamma}\left\{h^{r}(x, u(x))-g^{r}(x)\right\} \varphi^{r}(x) d x^{\prime} .
\end{gathered}
$$


Let us now formulate the regularity of the problem and the Liouville conditions.

Definition 2.1 $(R)$. We say that the problem (2.1), (2.4) is regular (and denote this property by $(R)$ ) if for each weak solution $u$ of this problem for which $D u \in L_{\infty}(\Omega)$, the gradient $D u$ is locally $\alpha$-Hölder continuous on $\Omega \cup \Gamma$, and for each $\Omega^{\prime}$ for which $\Omega^{\prime} \subset \Omega \cup \Gamma$ it holds

$$
\|\nabla u\|_{C^{\alpha}\left(\overline{\Omega^{\prime}}\right)} \leqq C,
$$

where the constant $C$ depends on $\|\nabla u\|_{L_{\infty}(\Omega)}, \Omega^{\prime}$ and the data of the problem.

Definition 2.2 $(L)$. We say that the system (2.1) satisfies the Liouville condition $(L)$ if for each $x_{0} \in \Omega$ and each $\xi \in \boldsymbol{R}^{m}$ the solution $u \in W_{\text {loc }}^{1,2}\left(\boldsymbol{R}^{n}\right)$ of the equation

$$
\int_{R^{n}}\left(a\left(x_{0}, \xi, D u\right), D \varphi\right) d x=0 \quad \forall \varphi \in C_{0}^{\infty}\left(R^{n}\right)
$$

for which $D u \in L_{\infty}\left(\boldsymbol{R}^{n}\right)$ is a polynomial of at most the first degree.

Definition 2.3 $\left(L^{+}\right)$. Write $Z=\left\{\varphi \in C_{0}^{\infty}\left(\boldsymbol{R}^{n}\right) ; \boldsymbol{C} \varphi=0\right.$ on $\left\{x \in \boldsymbol{R}^{n}\right.$; $\left.\left.x_{n}=0\right\}\right\}$. We say that the problem (2.1), (2.4) satisfies the Liouville condition $\left(L^{+}\right)$if for each $x_{0} \in \Gamma ; \xi \in \boldsymbol{R}^{m} ; d \in \boldsymbol{R}^{m}$ the solution $u \in W_{\text {loc }}^{1,2}\left(\overline{\boldsymbol{R}_{+}^{n}}\right)$ of the equation

$$
\int_{R_{+}^{n}}\left(a\left(x_{0}, \xi, D u\right), D \varphi\right) d x=\int_{\left.\mid x \in R^{n} ; x_{n}=0\right\}}(d, \varphi) d x^{\prime} \quad \forall \varphi \in Z
$$

is the polynomial of at most the first degree, provided that $C u$ is a polynomial of at most the first degree on $\left\{x \in \boldsymbol{R}^{n} ; x_{n}=0\right\}$ and $D u \in L_{\infty}\left(\boldsymbol{R}_{+}^{n}\right)$.

Our paper contains the proof that (roughly speaking): (2.1), (2.4) is regular iff $(L)$ and $\left(L^{+}\right)$hold simultaneously. The necessity of the Liouville conditions is proved in $\S 3$ with the definition of the regularity being slightly changed. In $\S 4$ the proof of the implication $(L) \wedge\left(L^{+}\right) \Rightarrow(R)$ is given.

3. The necessity of Liouville conditions. Considering the definitions 2.1-2.3 we conclude that the property $(R)$ concerns one fixed problem (2.1), (2.4) whilst the Liouville conditions $(L)$ and $\left(L^{+}\right)$ refer to a system of problems (2.8). We do not know whether the implication $(R) \Rightarrow(L) \wedge\left(L^{+}\right)$holds. To obtain the implication of this type we modify at first the definition of regularity. 
Definition $3.1\left(R^{\prime}\right)$. Let for each $x_{0} \in \overline{\boldsymbol{R}_{+}^{n}}, \xi \in \boldsymbol{R}^{m}, d \in \boldsymbol{R}^{m}$ and for each solution $u$ of (2.8) for which $D u \in L_{\infty}\left(R_{+}^{n}\right)$ and $C u$ is a polynomial of at most the first degree on $\left\{x \in \boldsymbol{R}^{n} ; x_{n}=0\right\}$ there exists $T>0$ such that $u$ belongs to the space $C^{1, \alpha}(\overline{B(0, T)})$ with $\alpha=$ $\min \{1 / 2,1-n / p\}$ and

$$
\|u\|_{C^{1, \alpha}} \leqq C,
$$

where $C$ and $T$ depend only on $\|D u\|_{L_{\infty}},|u(0)|$ and the data of the problem.

\section{THEOREM 3.1. $\left(R^{\prime}\right) \Rightarrow\left(L^{+}\right)$.}

Proof. Suppose $x_{0}=0$. The function $u_{R}(y)=(1 / R) u(R y)$ solves (2.8). Further $\left\|D u_{R}\right\|_{L_{\infty}}=\|D u\|_{L_{\infty}}$ and for $R>1$ the values $\left|u_{R}(0)\right|$ and $C u_{R}$ are bounded by the same constants as the corresponding values of $u$. Thus $u_{R}(R>1)$ satisfies (3.1) with the constant independent of $R$. Let $x \in \boldsymbol{R}_{+}^{n} ; T R \geqq|x| ; R y=x$. According to (3.1) we get

$$
\left|D_{y} u_{R}(y)-D_{y} u_{R}(0)\right| \leqq C|y|^{\alpha} \leqq C\left|\frac{x}{R}\right|^{\alpha}
$$

hence

$$
\left|D_{x} u(x)-D_{x} u(0)\right| \leqq C \frac{|x|^{\alpha}}{R^{\alpha}}
$$

and it implies that $D_{x} u(x)=D_{x} u(0)$ for $R \rightarrow \infty$.

Let us mention that the necessity of the condition $(L)$ was proved in [4].

4. Sufficiency of the Liouville conditions. Put for an arbitrary vector function $f=\left\{f^{r}\right\}_{r=1, \cdots, s}$,

$$
\left\{\begin{array}{c}
F\left(x_{0}, R\right)=R^{2-n} \int_{B\left(x_{0}, R\right)} \sum_{r=1}^{s} \sum_{i=1}^{n}\left|D_{i} f^{r}(x)\right|^{2} d x \text { and } \\
V B\left(x_{0}, R\right)=\left\{u \in W^{1,2}\left(B\left(x_{0}, R\right)\right) ; C u=0 \text { on } \Gamma\left(x_{0}, R\right)\right. \text { and } \\
\left.u=0 \text { on } \partial B\left(x_{0}, R\right) \backslash \Gamma\left(x_{0}, R\right)\right\} .
\end{array}\right.
$$

The following notation will be used in Lemma 4.1 only:

$$
B\left(x_{0}, t\right)=\left\{x \in \boldsymbol{R}_{+}^{n} ;\left|x-x_{0}\right|<t\right\} ; \quad t \in[0,1] .
$$

Let

$$
\Gamma=\left\{x \in \boldsymbol{R}^{n} ;\left|x^{\prime}\right|<1 ; x_{n}=0\right\} .
$$

With the so defined $B\left(x_{0}, t\right)$ the symbols $F\left(x_{0}, t\right)$ and $V B\left(x_{0}, t\right)$ have 
the same meaning as in (4.1).

LEMMA 4.1. Let $\boldsymbol{B}=\left\{B_{\substack{r s \\ i, j, j=1, \ldots, m \\ r, j, 1, \ldots, n}}^{i j}\right.$ be a real matrix such that

$$
\exists \mathscr{H}>0 \quad \forall \eta \in R^{n m} \quad B_{r s}^{i j} \eta_{r}^{i} \eta_{s}^{j} \geqq \mathscr{C}|\eta|^{2} .
$$

Then there exists a positive number $K$ such that for every $x_{0}=$ $(0, \cdots, 0, q),(q \in[0,1])$ for each $v \in W^{1,2}\left(B\left(x_{0}, 1\right)\right)$ for which

$$
C v=0 \text { on } \Gamma
$$

and which solves the system

$$
\int_{B\left(x_{0}, 1\right)}(B D v, D \varphi) d x=0 \quad \forall \varphi \in V B\left(x_{0}, 1\right),
$$

and for every $t \in] 0,1 / 2[$, the inequalities

$$
\begin{gathered}
V\left(x_{0}, t\right) \leqq K t^{2} V\left(x_{0}, 1\right) \\
t^{-n} \int_{B\left(x_{0}, t\right)}\left|v(x)-P_{t}\right|^{2} d x \leqq K t^{2} \int_{B\left(x_{0}, 1\right)}|v(x)-Q|^{2} d x
\end{gathered}
$$

hold, where $Q$ is an arbitrary vector such that $C Q=0$ and $P_{t}$ is either a value $v(\widetilde{x})$ at an arbitrary point $\tilde{x} \in \overline{B\left(x_{0}, t\right)}$ or an integral mean value of $v$ over any connected subset of $\overline{B\left(x_{0}, t\right)}$.

Proof. Let $k \in N$ be such that $W^{2, k}(G) \subset C^{1}(\bar{G})$ for a bounded domain $G \subset \boldsymbol{R}^{n}$. Let $1=t_{0}>t_{1}>>t_{k}=1 / 2$ be an equidistant subdivision of the segment $[1 / 2,1]$.

Let $\Phi \in C^{\infty}\left(\overline{B\left(x_{0}, 1\right)}\right) ; \operatorname{supp} \Phi \subset B\left(x_{0},\left(t_{0}+t_{1}\right) / 2\right) ; 0 \leqq \Phi \leqq 1, \Phi \equiv 1$ on $B\left(x_{0}, t_{1}\right) ;|D \Phi| \leqq C /\left(t_{0}-t_{1}\right)$.

Let $C Q=0$ and put

$$
\varphi=\Phi^{2}(v-Q)
$$

in (4.4). By usual calculations we obtain (denoting in what follows all the constants by $C$ )

$$
\int_{B\left(x_{0}, t_{1}\right)}|D v|^{2} d x \leqq C \int_{B\left(x_{0}, 1\right)}|v-Q|^{2} d x
$$

If $\overline{B\left(x_{0}, t_{1}\right)} \subset B\left(x_{0}, 1\right)$ we use the fact that all the derivatives up to the order $k$ solve the system (4.4) and we get finally the estimate

$$
\int_{B\left(x_{0}, 1 / 2\right)}\left|D^{k} v\right|^{2} d x \leqq C \int_{B\left(x_{0}, 1\right)}|v-Q|^{2} d x .
$$

If $B\left(x_{0}, t_{1}\right)$ reaches up to the boundary, only the tangent derivatives $D_{j} v(j=1, \cdots, n-1)$ of the solution $v$ solve again the boundary 
value problem. For them we get

$$
\int_{B\left(x_{0}, t_{2}\right)}\left|D\left(D_{j} v\right)\right|^{2} d x \leqq C \int_{B\left(x_{0}, t_{1}\right)}|D v|^{2} d x
$$

The second normal derivative can be expressed from the equation

$$
B_{r s}^{i j} D_{i j} v^{s}=0 ; \quad r=1, \cdots, m,
$$

which holds a.e. in $B\left(x_{0}, 1\right)$. Advancing this process up to the estimate of the derivatives of the $k$ th order we obtain (using the Sobolev imbedding theorem)

$$
\max ^{2}\left\{|D v(x)| ; x \in B\left(x_{0}, \frac{1}{2}\right)\right\} \leqq C \int_{B\left(x_{0}, 1\right)}|v-Q|^{2} d x
$$

Let now $t \in] 0,1 / 2], x, \widetilde{x} \in \overline{B\left(x_{0}, t x\right)}$. Then

$$
|v(x)-v(\widetilde{x})|^{2} \leqq C t^{2} \max ^{2}\left\{|D v(x)| ; x \in \overline{B\left(x_{0}, \frac{1}{2}\right)}\right\}=C \int_{B\left(x_{0}, 1\right)}|v-Q|^{2} d x .
$$

Let us recall that the constant $C$ does not depend on the position of the point $x_{0}$ satisfying the assumptions of Lemma 4.1. Its value will be needed in the next text; because of an easier quotation we denote it by $\mathrm{K}$. Integrating the last inequality over $B\left(x_{0}, t\right)$, we get (4.6) for the case $P_{t}=v(\widetilde{x})$ with $\widetilde{x} \in B\left(x_{0}, t\right)$. The case of $P_{t}$ being an integral mean value can be reduced to the previous one by means of the integral mean value theorem.

To prove the inequality (4.5) we start with the estimate $\left(4.8^{\prime}\right)$ and applying the same method as before, we obtain

$$
\left(\max \left\{|D v(x)| ; x \in \overline{B\left(x_{0}, \frac{1}{2}\right)}\right\}\right)^{2}=C \int_{B\left(x_{0}, 1\right)}|D v|^{2} d x .
$$

The inequality (4.5) is an immediate consequence of (4.10).

The main result of this section is the following

TheOREM 4.2. Let $(L)$ and $\left(L^{+}\right)$be satisfied. Let $u \in W^{1,2}(\Omega)$ with the gradient $D u \in L_{\infty}(\Omega)$ be a weak solution of the problem (2.1), (2.4). Then $D u$ is $\alpha$-Hölder continuous on $\Omega \cup \Gamma$ with $\alpha=$ $\min (1 / 2,1-n / p)$ and for every domain $\Omega^{\prime}$ such that $\bar{\Omega}^{\prime} \subset \Omega \cup \Gamma$ the inequality holds:

$$
\begin{gathered}
\|u\|_{C^{1, \alpha}\left(\bar{\Omega}^{\prime}\right)} \leqq C\left(\|D u\|_{L_{\infty},},\left\|u_{0}\right\|_{W^{2}, p},\|f\|_{W^{1, p / 2}},\right. \\
\left.\|g\|_{L_{\infty}}, \operatorname{dist}\left(\bar{\Omega}^{\prime}, R_{+}^{n} \backslash \Omega\right)\right) .
\end{gathered}
$$

Schema of the proof of the Theorem 4.2. In Lemma 4.8 we shall prove that $D u$ belongs to certain Morrey-Campanato space and use then embedding of this space into $C^{1, \alpha}$. 
For the case $\bar{\Omega}^{\prime} \subset \Omega$ it follows from the condition $(L)$. We can prove it by the method described in [4] modifying it slightly.

For the case $\bar{\Omega}^{\prime} \cap \Gamma \neq \varnothing$ more substantial modifications of the method are needed. Denoting tangent derivatives as $\omega_{l}^{r}=D_{l} u^{r}$; $r=1, \cdots, m ; l=1, \cdots, n-1$, we decompose them on $B(x, R)$ as

$$
\omega=v+w
$$

in the following way:

(i) The function $w$ solves the linearized equation in variations (see (4.14)) and satisfies the nonhomogeneous boundary conditions

$$
\begin{aligned}
& C w_{l}=C D_{l} u_{0} \quad \text { on } \quad \Gamma(x, R), \\
& w_{l}-D_{l} u_{0}=0 \quad \text { on } \quad \partial B(x, R) \backslash \Gamma(x, R),
\end{aligned}
$$

$l=1, \cdots, n-1$. The $L_{2}$-norm of $D w$ can be easily estimated (see Lemma 4.3).

(ii) The second component $v=\omega-w$ solves the homogeneous linearized equation (4.15) and satisfies the homogeneous boundary conditions $C v_{l}=0$ on $\Gamma(x, R)$ and nonhomogeneous boundary conditions $v_{l}=\omega_{l}-D_{l} u_{0}$ on $\partial B(x, R) \backslash \Gamma(x, R), l=1, \cdots, n-1$.

In Lemma 4.4 we shall prove that, starting with sufficiently small oscillations of $v$ on $B(x, R)$ we can describe how they decrease on $B(x, \tau R),(\tau \in(0,1))$.

The Liouville condition $\left(L^{+}\right)$yields, for each $x_{0} \in \Gamma$, the fact that

$$
\liminf _{R \rightarrow 0_{+}} V\left(x_{0}, R\right)=0 . \quad \text { (See Theorem 4.5.) }
$$

Combining this result together with the estimates of $v$ and $w$, we obtain the assertion of Theorem 4.2.

First we shall describe more precisely the decomposition of $\omega$.

Let $u$ be a solution of (2.6) with $D u \in L_{\infty}(\Omega)$. Using the finite difference technique, we prove that $u \in W_{\text {loc }}^{2,2}(\Omega)$ and that each component $\omega_{l}$ of the tangent gradient $\omega$ solves the equation

$$
\begin{aligned}
\int_{\Omega}\{( & \left.\frac{\partial a}{\partial \eta} D \omega_{l}+\frac{\partial a}{\partial \xi} \omega_{l}+\frac{\partial a}{\partial x_{l}}, D \varphi\right)+\left(\frac{\partial \bar{a}}{\partial \eta} D \omega_{l}\right. \\
& \left.\left.+\frac{\partial \bar{a}}{\partial \xi} \omega_{l}+\frac{\partial \bar{a}}{\partial x_{l}}, \varphi\right)\right\} d x=\int_{\Omega}\left(\frac{\partial f}{\partial x_{l}}, \varphi\right) d x \\
& +\int_{I}\left\{\left(\frac{\partial h}{\partial \xi} \omega_{l}+\frac{\partial h}{\partial x_{l}}-\frac{\partial g}{\partial x_{l}}, \varphi\right)\right\} d x^{\prime}, \quad \forall \varphi \in V .
\end{aligned}
$$

Moreover, $C\left(\omega_{l}-D_{l} u_{0}\right)=0$ on $\Gamma$.

Let $x_{0} \in \Omega \cup \Gamma ; R>0$ and $x_{0 n} \leqq R$ (i.e., $\Gamma\left(x_{0}, R\right) \neq \varnothing$ ). Define $w=\left\{w^{r}\right\}_{r=1, \cdots, m} \in W^{1,2}\left(B\left(x_{0}, R\right)\right)$ as a unique weak solution of the problem 


$$
w_{l}-D_{l} u_{0} \in V B\left(x_{0}, R\right) \text {, }
$$

$\forall \varphi \in V B\left(x_{0}, R\right)$

$$
\begin{aligned}
\int_{B\left(x_{0}, R\right)} & \left\{\left(\frac{\partial a}{\partial \eta}(x, u, D u) D w_{l}, D \varphi\right)+\left(\frac{\partial \bar{a}}{\partial \eta}(x, u, D u) D w_{l}, \varphi\right)\right\} d x \\
= & -\int_{B\left(x_{0}, R\right)}\left\{\left(\frac{\partial a}{\partial \xi} \omega_{l}+\frac{\partial a}{\partial x_{l}}, D \varphi\right)+\left(\frac{\partial \bar{a}}{\partial \xi} \omega_{l}+\frac{\partial \bar{a}}{\partial x_{l}}, \varphi\right) d x\right. \\
& +\int_{B\left(x_{0}, R\right)}\left(\frac{\partial f}{\partial x_{l}}, \varphi\right) d x+\int_{\Gamma\left(x_{0}, R\right)}\left\{\frac{\partial h}{\partial \xi} \omega_{l}+\frac{\partial h}{\partial x_{l}}+\frac{\partial g}{\partial x_{l}}, \varphi\right\} d x^{\prime} .
\end{aligned}
$$

The relations (4.12) and (4.13), (4.14) imply that (defining $v_{l}=\omega_{l}-w_{l}$ ) the component $v_{l}$ solves the equation

$\forall \varphi \in V B\left(x_{0}, R\right)$

$$
\int_{B\left(x_{0}, R\right)}\left\{\left(\frac{\partial a}{\partial \eta}(x, u, D u) D v_{l}, D \varphi\right)+\left(\frac{\partial \bar{a}}{\partial \eta}(x, u, D u) D v_{l}, \varphi\right) d x=0,\right.
$$

and satisfies the boundary conditions

$$
\begin{aligned}
C v_{l} & =0 \text { on } \Gamma\left(x_{0}, R\right) \\
v_{l} & =\omega_{l}-D_{l} u_{0} \text { on } \partial B\left(x_{0}, R\right) \backslash \Gamma\left(x_{0}, R\right) .
\end{aligned}
$$

The components $v_{l}$ and $w_{l}$ depend on the choice of $x_{0}$ and $R$. We shall denote them by $v=\left\{v_{l}\right\}_{l=1, \cdots, n-1}$, omitting to express the dependence on $x_{0}$ and $R$ if not necessary.

Taking into account the assumptions on the coefficients, the right-hand side, the boundary conditions, and the solution $u$ ( $D u \in$ $L_{\infty}(\Omega)$ ), we get easily that the problem (4.13), (4.14) can be rewritten as follows:

$$
\begin{gathered}
w-w_{0} \in V B\left(x_{0}, R\right) ; \\
\int_{B\left(x_{0}, R\right)}\{(\boldsymbol{A} D w, D \varphi)+(\overline{\boldsymbol{A}} D w, \varphi)\} d x \\
=\int_{B\left(x_{0}, R\right)}(\boldsymbol{F}, \varphi) d x+\int_{\Gamma^{\prime}\left(x_{0}, R\right)}(\boldsymbol{H}, \varphi) d x^{\prime} \quad \forall \varphi \in V B\left(x_{0}, R\right),
\end{gathered}
$$

where

$$
\begin{array}{ll}
\text { (1) } & \boldsymbol{A}=\left\{\frac{\partial a_{i}^{r}}{\partial \eta_{j}^{s}} \delta_{k l}\right\} \in L_{\infty}\left(B\left(x_{0}, R\right)\right), \\
& (\boldsymbol{A} \eta, \eta) \geqq \mathscr{H}|\eta|^{2} \quad \forall \eta \in R^{n m(n-1)}, \\
\text { (2) } & \overrightarrow{\boldsymbol{A}}=\left\{\frac{\partial \bar{a}^{r}}{\partial \eta_{j}^{s}}\right\} \in L_{\infty}\left(B\left(x_{0}, R\right)\right), \\
\text { (3) } w_{0}=D_{l} u_{0} \in W^{1, p}\left(B\left(x_{0}, R\right)\right) \\
\text { (4) } \boldsymbol{F} \in L_{p / 2}\left(B\left(x_{0}, R\right)\right) \\
\text { (5) } \boldsymbol{H} \in L_{\infty}\left(\Gamma\left(x_{0}, R\right)\right) .
\end{array}
$$


(Let us remind here that $\omega_{l}$ is bounded on $\Omega$ and (as it solves the system 4.12) it belongs to the space $W_{1 \text { loc }}^{1,2}(\Omega \cup \Gamma)$; thus it has a well defined trace on $\Gamma$ and, since $\|D u\|_{L_{\infty}} \leqq C$, we have $\left\|\omega_{l}\right\|_{L_{\infty}(\Gamma)} \leqq C$, too.)

Putting $\varphi=w-w_{0}$ and using the assumptions (4.19), we get

LEMMA 4.3. There exist a positive constant $C$ and $a$ positive radius $R_{0}$ such that, for every $R \in\left(0, R_{0}\right)$ and for every solution $w \in W^{1,2}\left(B\left(x_{0}, R\right)\right)$ of the problem (4.17), (4.18) satisfying (4.19), the inequality

$$
\|D w\|_{L_{2}\left(B\left(x_{0}, R\right)\right)} \leqq C R^{n(1 / 2-1 / p)}
$$

holds.

The local behaviour of the oscillations of the second component $v$ is shown in the next lemma.

Lemma 4.4. For every $\Omega^{\prime} ; \bar{\Omega}^{\prime} \subset \Omega \cup \Gamma$, for every positive $C$ and each $\tau \in(0,1)$, there exist a positive $\varepsilon$ and $R_{0}$ such that, for every solution $u$ of the problem (2.6) with $\|D u\|_{L_{\infty}} \leqq C$, for every $x_{0} \in \Omega^{\prime}$ and $R \in] 0, \min \left(R_{0}, 1-\left|x_{0}\right|\right)[$, the implication

$$
V\left(x_{0}, R\right)<\varepsilon^{2} \Longrightarrow V\left(x_{0}, \tau R\right)<2 K \tau^{2} V\left(x_{0}, R\right)
$$

holds.

(Here

$$
V\left(x_{0}, R\right)=R^{2-n} \int_{B\left(x_{0}, R\right)}|D v|^{2} d x, \quad V\left(x_{0}, \tau R\right)=(\tau R)^{2-n} \int_{B\left(x_{0}, \tau R\right)}|D v|^{2} d x
$$

and, in both the expressions, $v$ is the component of the decomposition of $\omega$ on $B\left(x_{0}, R\right)$ ). $K$ is the maximal of the constants from Lemma 4.1 , corresponding to

$$
B_{i j}^{r s}=\frac{\partial a_{i}^{r}}{\partial \xi_{j}^{s}}\left(x_{0}, \zeta, \xi\right),
$$

$x_{0} \in \bar{\Omega} ;|\xi| \leqq C$ and the upper bound for $\zeta$ derived as the upper bound for weak solution of the problem (2.6) for which $\|D u\|_{L_{\infty}(\Omega)} \leqq C$.

Proof. Suppose that the assertion of the lemma does not hold. Then there exist $C \in(0, \infty), \tau \in] 0,1\left[\right.$, sequences $\varepsilon_{\nu} \searrow 0, R_{\nu} \searrow 0, x_{\nu} \rightarrow$ $x_{0} \in \Omega \cup \Gamma$ and $u_{\nu} ;\left\|D u_{\nu}\right\|_{L_{\infty}} \leqq C$, such that

$$
V\left(x_{\nu}, R_{\nu}\right)=\varepsilon_{\nu}^{2}
$$

and simultaneously 


$$
V\left(x_{\nu}, \tau R_{\nu}\right)>2 K \tau^{2} V\left(x_{\nu}, R_{\nu}\right) .
$$

If $x_{0} \in \Omega$, all $\overline{B\left(x_{\nu}, R_{\nu}\right)} \subset \Omega$ for a sufficiently large and the proof is substantially the same as in [4]. A similar situation occurs if $x_{0} \in \Gamma$ but $R_{\nu}<x_{\nu n}$ for infinitely many indices $\nu$ (i.e. the closed sets $\left.\overline{B\left(x_{\nu}, R_{\nu}\right)} \subset \Omega\right)$. In what follows, $x_{\nu j}$ will denote the $j$ th component of the vector $x_{\nu}$ (i.e., $x_{\nu}=\left\{x_{\nu j}\right\}_{j=0}^{m}$ ). The same notation will be used for sequences $u_{\nu}, v_{\nu}$ etc.

Suppose that $x_{\nu} \rightarrow x_{0} \in \Gamma$ and $x_{\nu n} \leqq R_{\nu}$. Using the decomposition $\omega_{\nu l}=w_{\nu l}+v_{\nu l}$ on $B\left(x_{\nu}, R_{\nu}\right)$ and estimating $D v_{\nu}$ by (4.22) and $D w_{\nu}$ by Lemma 4.3 we get

$$
\left\|D \omega_{\nu l}\right\|_{L_{2}\left(B\left(x_{\nu}, R_{\nu}\right)\right)}^{2} \leqq c\left(R_{\nu}^{n(1-2 / p)}+\varepsilon_{\nu}^{2} R_{\nu}^{n-2}\right), \quad l=1, \cdots, n-1 .
$$

The second normal derivatives $\partial^{2} u_{\nu} / \partial x_{n}^{2}$ can be expressed from the equation

$$
\frac{\partial a_{i}^{r}}{\partial \eta_{j}^{s}} D_{i+j} u^{s}+\frac{\partial a_{i}^{r}}{\partial \xi_{s}} D_{i} u^{s}+\frac{\partial a_{i}^{r}}{\partial x_{i}}+\bar{a}^{r}-f^{r}=0 ; \quad r=1, \cdots, m,
$$

which is satisfied a.e. on $\Omega$. Thus we get

$$
\left\|D^{2} u_{\nu}\right\|_{L_{2}\left(B\left(x_{\nu}, R_{\nu}\right)\right)}^{2} \leqq C\left(R_{\nu}^{n(1-2 / p)}+\varepsilon_{\nu}^{2} R_{\nu}^{n-2}\right) .
$$

Put

$$
\begin{gathered}
\mathscr{H}_{\nu l}=\frac{1}{\operatorname{meas}_{n-1} \Gamma\left(x_{\nu}, R_{\nu}\right)} \int_{\Gamma\left(x_{\nu}, R_{\nu}\right)} v_{\nu l}(y) d y^{\prime}, \\
\mathscr{H}_{\nu}=\left\{\mathscr{H}_{\nu l}\right\}_{l=1, \cdots, n-1}, \\
\psi_{\nu}: y \longrightarrow x,
\end{gathered}
$$

where

$$
\begin{aligned}
& x_{i}=x_{\nu i}+R_{\nu} y^{i} \quad i=1, \cdots, n-1, \\
& x_{n}=R_{\nu} y_{n},
\end{aligned}
$$

$$
a_{\nu}=1+\frac{x_{\nu n}}{R_{\nu}} \in\langle 1,2\rangle
$$

Then the substitution $\psi_{\nu}^{-1}$ transforms the $\operatorname{sets} B\left(x_{\nu}, R_{\nu}\right)$ into

$$
B_{\nu}=\left\{y \in \boldsymbol{R}^{n} ;\left|y_{i}\right|<1 \text { for } i=1, \cdots, n-1,0<y_{n}<a_{\nu}\right\}
$$

and the sets $\Gamma\left(x_{\nu}, R_{\nu}\right)$ into

$$
\Gamma_{0}=\left\{y \in \boldsymbol{R}^{n} ;\left|y_{i}\right|<1 \text { for } i=1, \cdots, n-1, y_{n}=0\right\} .
$$

Moreover, put

$$
B_{\nu, \tau}=\psi_{\nu}^{-1}\left(B\left(x_{\nu}, \tau R_{\nu}\right)\right)
$$


Defining

$$
s_{\nu}(y)=\frac{1}{\varepsilon_{\nu}}\left\{v_{\nu}\left(\psi_{\nu}(y)\right)-\mathscr{C}_{\nu}\right\},
$$

we get from (4.22), (4.23) that

$$
\begin{gathered}
S_{\nu}=\int_{B_{\nu}}\left|D s_{\nu}\right|^{2} d y=1, \\
S_{\nu, \tau}=\int_{B_{\nu, \tau}}\left|D s_{\nu}\right|^{2} d y>2 K \tau^{2} .
\end{gathered}
$$

Applying the following type of Poincarés inequality to $s_{\nu}$ and using (4.32), we obtain

$$
\left\|s_{\nu}\right\|_{W^{1,2}\left(B_{\nu}\right)} \leqq C
$$

Poincaré's inequality. There exists $C>0$ such that for each $\nu \in N$ and for each $f \in W^{1,2}\left(B_{\nu}\right)$

$$
\int_{B_{\nu}}\left[f(y)-\frac{1}{\operatorname{meas}_{n-1} \Gamma_{0}} \int_{\Gamma_{0}} f(z) d z^{\prime}\right]^{2} d y \leqq C \int_{B_{\nu}}|D f|^{2} d y
$$

holds.

In what follows, we dare to pass to a suitable subsequence without notice and without changing the notation.

We distinguish two cases

$$
\begin{aligned}
& \text { (a) } a_{\nu} \searrow a_{0} ; \bigcap_{\nu \in N} B_{\nu} \supset B_{0} \\
& \quad=\left\{y \in R^{n} ;\left|y_{i}\right|<1 ; i=1, \cdots, n-1,0<y_{n}<a_{0}\right\} ; \\
& \text { (b) } a_{\nu} \nearrow a_{0} ; \bigcup_{\nu \in N} B_{\nu}=B_{0} .
\end{aligned}
$$

We shall prove that $\left\{s_{\nu}\right\}$ converges on $B_{0}$ to a function $s$ solving the system with constant coefficients and such boundary conditions that Lemma 4.1 can be applied to $s$. Then the passage to the limit in the relations (4.32), (4.33) gives the contradiction.

From (4.34) we can conclude that there is a function $s \in W^{1,2}\left(B_{0}\right)$ such that

$$
s_{\nu} \longrightarrow s \text { and } \varepsilon_{\nu} s_{\nu} \longrightarrow 0 \text { a.e. on } B_{0}
$$

and

(a) $s_{y} \longrightarrow s$ in $W^{1,2}\left(B_{0}\right)$, weakly

(b) $s_{\nu} \longrightarrow s$ in $W^{1,2}\left(G_{0}\right)$ weakly for each

$$
\dot{G} ; \bar{G} \subset \bar{B}_{0} \mid\left\{y \in \boldsymbol{R}^{n} ; y_{n}=a_{0}\right\} \text {. }
$$

Taking into account the definition of $s_{\nu}$ (see (4.31)), we get 


$$
\omega_{\nu}\left(\psi_{\nu}(y)\right)=\varepsilon_{\nu} s_{\nu}(y)+\mathscr{H}_{\nu}+t_{\nu}(y),
$$

where

$$
t_{\nu}(y)=w_{\nu}\left(\psi_{\nu}(y)\right)
$$

The boundedness of $\omega$ together with Lemma 4.3 and (4.37) yield the

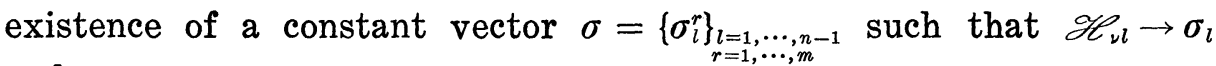
and

$$
\omega_{\nu l}\left(\psi_{\nu}(y)\right) \longrightarrow D_{l} u_{0}\left(x_{0}\right)+\sigma_{l} \quad \text { a.e. on } B_{0} \text {. }
$$

A similar technique may be used for the normal derivative. Put

$$
\mathscr{C}_{\nu n}=\frac{1}{\operatorname{meas}_{n-1} \Gamma\left(x_{\nu}, R_{\nu}\right)} \int_{\Gamma\left(x_{\nu}, R_{\nu}\right)} D_{n} u_{\nu}\left(x^{\prime}, x_{n}\right) d x^{\prime} .
$$

By Poincaré's inequality and (4.25) we get

$$
\left\|D_{n} u_{\nu}\left(\psi_{\nu}(y)\right)-\mathscr{H}_{\nu n}\right\|_{L_{2}\left(B_{\nu}\right)}^{2} \leqq C\left(R_{\nu}^{2(1-n / p)}+\varepsilon_{\nu}^{2}\right) \longrightarrow 0 \text {. }
$$

This and the boundedness of $D u$ imply the boundedness of the sequence $\mathscr{H}_{\nu n}$ and thus the existence of such a constant vector $\xi_{n}=$ $\left\{\xi_{n}^{r}\right\}_{r=1, \cdots, m}$ that

$$
D_{n} u_{\nu}\left(\psi_{\nu}(y)\right) \longrightarrow \xi_{n} \quad \text { a.e. on } B_{0} \text {. }
$$

Put $\xi=\left\{\xi_{l}^{r}\right\}_{\substack{r=1, \ldots, m \\ l=1, \ldots, n}} ; \xi_{l}^{r}=D_{l} u_{0}^{r}\left(x_{0}\right)+\sigma_{l}^{r}$ for $r=1, \cdots, m$ and $l=1, \cdots$, $n-1$. Then $(4.40)$ and (4.42) give

$$
D_{l} u_{\nu}^{r}\left(\psi_{\nu}(y)\right) \longrightarrow \xi_{l}^{r} \text { a.e. on } B_{0} \text { for } r=1, \cdots, m, l=1, \cdots, n,
$$

and the norm of $\xi$ is bounded by the same constant as the $L_{\infty}$-norm of $D u_{\nu}$.

Deduce now the equation for $s$ :

Substituting $x=\psi_{\nu}(y)$ into (4.15) and using (4.31), we obtain

$$
\int_{B_{\nu}}\left\{\left(M D s_{\nu}, D \varphi\right)+R_{\nu}\left(\bar{M} D s_{\nu}, \varphi\right)\right\} d y=0 \quad \forall \nu \in N, \forall \varphi \in V B_{\nu} ;
$$

where

$$
\begin{array}{ll}
\boldsymbol{M}=\left\{m_{i j}^{r s}(\nu, y)\right\}_{\substack{r, s=1, \ldots, m \\
i, j=1, \cdots, n}}, & m_{i j}^{r s}(\nu, y)=\frac{\partial a_{i}^{r}}{\partial \eta_{j}^{s}}\left(\psi_{\nu}(y), u_{\nu}\left(\psi_{\nu}(y)\right), D u_{\nu}\left(\psi_{\nu}(y)\right)\right), \\
\overline{\boldsymbol{M}}=\left\{\bar{m}_{i j}^{r}(\nu, y)\right\}_{\substack{r=1, \ldots, m \\
i, j=1, \cdots, n}}, & \bar{m}_{i j}^{r}(\nu, y)=\frac{\partial \bar{a}^{r}}{\partial \eta_{j}^{s}}\left(\psi_{\nu}(y), u_{\nu}\left(\psi_{\nu}(y)\right), D u_{\nu}\left(\psi_{\nu}(y)\right)\right) .
\end{array}
$$

Taking into account that $\psi_{\nu}(y) \rightarrow x_{0}$ on $B_{0}, u_{\nu}\left(\psi_{\nu}(y)\right) \rightarrow \zeta$ on $B_{0}$ and $D u_{\nu}\left(\psi_{\nu}(y)\right) \rightarrow \xi$ a.e. on $B_{0}$, we can conclude that 


$$
m_{i j}^{r s}(\nu, y) \longrightarrow B_{i j}^{r s}=\frac{\partial a_{i}^{r}}{\partial \eta_{j}^{s}}\left(x_{0}, \zeta, \xi\right) \quad \text { a.e. on } B_{0}
$$

and passing to the limit in (4.44), we get finally

$$
\int_{B_{0}}(\boldsymbol{B} D s, D \varphi) d y=0 \quad \forall \varphi \in V B_{0} \bullet
$$

According to (4.16), $C v_{\nu}=0$ on $\Gamma\left(x_{\nu}, R_{\nu}\right)$ for each $\nu \in N$, hence $C s_{\nu}=0$ on $\Gamma_{0}$ for each $\nu \in N$ and

$$
C s=0 \text { on } \Gamma_{0} .
$$

Thus the function $s$ solves the boundary value problem of the type required in Lemma 4.1 and

$$
S_{\tau}=\tau^{2-n} \int_{B_{0, \tau}}|D s|^{2} d y \leqq K \tau^{2} \int_{B_{0}}|D s|^{2} d y=K \tau^{2} S,
$$

where $B_{0, \tau}=\left\{y \in \boldsymbol{R}^{n} ;\left|y_{i}\right|<\tau\right.$ for $\left.i=1, \cdots, n-1,\left|y_{n}-a_{0}+1\right|<\tau\right\}$, and $K$ is the constant described in Lemma 4.4 .

The weak lower semicontinuity of the functional $\vartheta: s \rightarrow \int_{B_{0}}|D s|^{2} d y$ together with (4.37), (4.32) gives

$$
S=\int_{B_{0}}|D s|^{2} d y \leqq 1
$$

To get the contradiction it is sufficient to prove that

$$
S_{\nu, \tau} \longrightarrow S_{\tau}=\tau^{2-n} \int_{B_{0, \tau}}|D s|^{2} d y
$$

We shall prove (by the choice of a test function) that $D s_{\nu} \rightarrow D s$ in $L_{2, \text { loc }}\left(B_{0}\right)$. Let us sketch the choice for the case (a): Take $\nu_{0} \in N$ so large that

$$
B_{\nu_{0}, \tau} \subset\left\{y \in \boldsymbol{R}^{n} ; y_{n}<a_{0}-1+\frac{\tau+1}{2}\right\} ;
$$

let $\Phi \in C_{\infty}\left(\bar{R}_{+}^{n}\right)$; supp $\Phi \subset B_{0} \cup \Gamma_{0} ; \Phi \equiv 1$ on $\bigcup_{\nu \geq \nu_{0}} B_{\nu, \tau} ;$ Then $\varphi=\left(s_{\nu}-s\right) \Phi^{2}$ (prolonged by zero if necessary) is an admissible test function for both (4.44) and (4.46). Therefore

$$
\begin{gathered}
\int_{B_{0}}\left\{\left(\boldsymbol{M} D s_{\nu}, D\left(s_{\nu}-s\right)\right) \Phi^{2}+2 \Phi\left(\boldsymbol{M} D s_{\nu},\left(s_{\nu}-s\right) D \Phi\right.\right. \\
\left.+\left(\bar{M} D s_{\nu}, s_{\nu}-s\right) \Phi^{2}\right\} d y=0 \\
\int_{B_{0}}\left\{\left(\boldsymbol{B} D s, D\left(s_{\nu}-s\right)\right) \Phi^{2}+2 \Phi\left(\boldsymbol{B} D s,\left(s_{\nu}-s\right)\right) D \Phi\right\} d y=0 .
\end{gathered}
$$

Finally, using the ellipticity condition 


$$
\begin{aligned}
\int_{B_{0}} \Phi^{2}\left|D\left(s_{\nu}-s\right)\right|^{2} d y \leqq \frac{1}{\mathscr{C}} \int_{B_{0}} \Phi^{2}\left(M D\left(s_{\nu}-s\right), D\left(s_{\nu}-s\right)\right) d y \\
\quad=\frac{1}{\mathscr{H}}\left\{\int_{B_{0}} \Phi^{2}\left(M D s_{\nu}, D\left(s_{\nu}-s\right)\right) d y-\int_{B_{0}} \Phi^{2}\left(M D s, D\left(s_{\nu}-s\right)\right) d y\right\} .
\end{aligned}
$$

Now we can estimate the first integral on the right hand side of (4.52) from (4.50) and the second one from (4.51) and we get

$$
\int_{B_{0}} \Phi^{2}\left|D\left(s_{\nu}-s\right)\right|^{2} d y \longrightarrow 0 \text {. }
$$

To bound the difference $S_{\nu, \tau}-S_{\tau}$ we write

$$
\begin{aligned}
\left|S_{\nu, \tau}-S_{\tau}\right| \leqq & \tau^{2-n}\left\{\int_{B_{0}} \Phi^{2}\left(D s_{\nu}^{2}-D s^{2}\right) d y+\int_{B_{0, \tau} \backslash B_{\nu, \tau}}|D s|^{2} d y\right. \\
& \left.+\int_{B_{\nu, \tau} \backslash B_{0, \tau}}|D s|^{2} d y\right\} .
\end{aligned}
$$

Here the first integral on the right hand side tends to zero by (4.53) and the second and third ones because of the uniform absolute continuity. Thus

$$
S_{\tau}=\lim _{\nu \rightarrow \infty} S_{\nu, \tau} \geqq 2 K \tau^{2} \geqq 2 K \tau^{2} S,
$$

which contradicts (4.48).

THEOREM 4.5. Let the system (2.1), (2.4) satisfies the condition $\left(L^{+}\right)$, let $u$ be a weak solution of (2.1), (2.4) for which $D u \in L_{\infty}(\Omega)$. Then for each $x_{0} \in \Gamma$, there exists a sequence $R_{\nu} \searrow 0$ such that

$$
\lim _{\nu \rightarrow \infty} Z\left(x_{0}, R_{\nu}\right)=0 \text {, }
$$

where $z=D u-D u_{0} . \quad\left(\right.$ For $Z\left(x_{0}, R_{\nu}\right)$ see $\left.(4.1)\right)$.

Proof. Be $x_{0} \in \Gamma ; 0<R<\operatorname{dist}\left(x_{0}, \partial \Omega \backslash \Gamma\right)$. Put

$$
\begin{gathered}
y=y(x)=\frac{x-x_{0}}{R}, \\
u_{R}(y)=\frac{u\left(x_{0}+R y\right)-u\left(x_{0}\right)}{R} .
\end{gathered}
$$

Then $y\left(B\left(x_{0}, R\right)\right)=B(0,1)$. Put $0_{R}=y(\Omega)$. For each $T$, let $R(T)$ be such a positive radius that it is $B(0, T) \subset 0_{R}$ for $R<R(T)$.

In the following part of the proof we use the fact that for every $T>0$ the set of second gradients $\left\{D^{2} u_{R} ; R<R(T)\right\}$ is bounded in $L_{2}(B(0, T))$. More precisely, it holds 
LEMMA 4.6. Let $u$ be a solution of the problem (2.1), (2.4) for which $D u \in L_{\infty}(\Omega)$. Then for each $x_{0} \in \Gamma$ and for every $T$, there exist $R(T)$ and $C$ such that

$$
\left\|D^{2} u_{R}\right\|_{L_{2}(B(0, T))} \leqq C \quad \forall R<R(T) .
$$

The value of $C$ depends on $\|D u\|_{L_{\infty}},\|f\|_{W^{1, p / 2}},\left\|u_{0}\right\|_{W^{2}, p},\|g\|_{W^{1, \infty}}, T$, and $\operatorname{dist}\left(x_{0}, \partial \Omega \backslash \Gamma\right)$.

Proof of the Lemma 4.6 is standard: using the finite difference technique and Nirenberg's lemma, we get the estimates for $D_{i j} u_{R}$, $i j \neq n n$. The bound for $D_{n n} u_{R}$ can be obtained by means of the equation in variations, which is valid a.e. on $B(0, T)$, and which enables us to express $D_{n n} u_{R}$ through the other second derivatives which we had estimated before.

Returning to the proof of Theorem 4.5, we see that the set $\left\{D u_{R} ; R<R(T)\right\}$ is bounded in $L_{\infty}(\Omega)$-it follows from the assumption $D u \in L_{\infty}(\Omega)$ and the simple equality

$$
\frac{\partial u_{R}(y)}{\partial y_{i}}=\frac{\partial u}{\partial x_{i}}\left(x_{0}+R y\right) \text {. }
$$

Taking into account that $u_{R}(0)=0$, we get finally the boundedness of the set $\left\{u_{R} ; R<R(T)\right\}$ in $W^{2,2}(B(0, T))$. The compactness of the imbedding of $W^{2,2}(B(0, T))$ into $W^{1,2}(B(0, T))$ allows us to choose a sequence $R_{\nu}, R_{\nu} \searrow 0$, such that $u_{R_{\nu}} \rightarrow p$ in $W^{1,2}(B(0, T)$ ), and, using the diagonal process, also

$$
\begin{aligned}
& \lim _{\nu \rightarrow \infty} u_{R_{\nu}}=p \quad \text { in } \quad W_{10 \mathrm{co}}^{1,2}\left(\boldsymbol{R}_{+}^{n}\right), \\
& \lim _{\nu \rightarrow \infty} D u_{R_{\nu}}=D p \quad \text { a.e. on } \boldsymbol{R}_{+}^{n} .
\end{aligned}
$$

Deduce now the equation for the limit function $p$ : To this end we substitute (4.56) and (4.57) into (2.6); after the passage to the limit we obtain

$$
\int_{\boldsymbol{R}_{+}^{n}}\left(a\left(x_{0}, \xi, D p(y)\right), D \varphi(y)\right) d y=\int_{\left\{y \in \boldsymbol{R}^{n} ; y_{n}=0\right\}}(d, \varphi(y)) d y^{\prime} .
$$

Using the theorem on traces and (4.59), we get

$$
\lim _{\nu \rightarrow \infty} \boldsymbol{C} u_{R_{\nu}}=\boldsymbol{C p} \quad \text { a.e. on } \quad\left\{y \in \boldsymbol{R}^{n} ; y_{n}=0\right\}
$$

The transformed boundary conditions give

$$
C u_{R, v}=C\left(\frac{u_{0}\left(x_{0}+R_{\nu} y\right)-u_{0}\left(x_{0}\right)}{R_{\nu}}\right),
$$


but $u_{0} \in C_{1}(\bar{\Omega})$, hence

$$
u_{0, R_{\nu}}=R_{\nu}^{-1}\left(u_{0}\left(x_{0}+R_{\nu} y\right)-u_{0}\left(x_{0}\right)\right) \longrightarrow y_{1} D_{1} u_{0}\left(x_{0}\right)+\cdots+y_{n} D_{n} u_{0}\left(x_{0}\right),
$$

so that $\boldsymbol{C p}$ is a polynomial of at most the first degree on $\left\{y \in \boldsymbol{R}^{n}\right.$; $y_{n}=0$. The condition $\left(L^{+}\right)$implies that $p$ is a polynomial of at most the first degree on $\boldsymbol{R}_{+}^{n}$.

Because of (4.59) and the fact that $D p$ is a constant vector, we have that

$$
D\left(x_{0}, R_{\nu}\right)=\int_{B(0,1)}\left|D u_{R_{\nu}}(y)-\left(D u_{R_{\nu}}\right)_{0,1}\right|^{2} d y \longrightarrow 0
$$

here $(D u)_{0,1}$ is the integral mean value of $D u$, i.e.

$$
(D u)_{0,1}=\frac{1}{\operatorname{meas}_{n} B(0,1)} \int_{B(0,1)} D u d y .
$$

After an easy calculation $\left(u_{0} \in W^{2, p}\right.$ with $\left.p>n\right)$ we obtain that also

$$
\begin{aligned}
\widetilde{Z}\left(x_{0}, R\right) & =\int_{B(0,1)} \mid D\left[u_{R_{\nu}}-u_{0, R_{\nu}}\right](y) \\
& -\left.\left(D\left[u_{R_{\nu}}-u_{0, R_{\nu}}\right]\right)_{0,1}\right|^{2} d y \longrightarrow 0 .
\end{aligned}
$$

The following lemma shows the relations between $Z$ and $\tilde{Z}$.

LEMMA 4.7. Let the notation of the preceding lemma be preserved. Then there exists constants $\gamma>0, \gamma_{1}>0$ such that for each point $x_{0} \in \Gamma, R<\operatorname{dist}\left(x_{0}, \partial \Omega \backslash \Gamma\right)$, the estimate

$$
Z\left(x_{0}, \frac{R}{2}\right) \leqq \gamma \widetilde{Z}\left(x_{0}, R\right)+\gamma_{1}\left\|D^{2} u_{0}\right\|_{L_{p}}^{2} R^{2(1-n / p)}
$$

holds.

The proof of this lemma is similar to that of Lemma 4.1 - we insert a suitable test function of the type $\Phi^{2}\left(\omega-\omega_{0}-c\right)\left(\right.$ here $\omega_{0}=$ $\left\{D_{i} u_{0}\right\}_{i=1, \cdots, n-1}$; $c$ is a constant vector satisfying the condition $C c=0$ ) into the equation in variations.

From (4.61) and (4.62) the assertion (4.55) of Theorem 4.5 follows.

To finish the proof of Theorem (4.2) it remains to observe that the difference between $Z\left(x_{0}, R\right)$ and $V\left(x_{0}, R\right)$ is small for small $R$ thanks to the assumption $u_{0} \in W^{2, p}(\Omega)$ and to use the same procedure as in [2], proof of Proposition 1.1 for the estimates of tangential derivatives. As for the second normal derivative, we repeat the estimates of (4.25). In such a way we get that the whole gradient belongs to the Morrey-Campanato space and thus $u \in C^{1, \alpha}\left(\overline{\left.B\left(x_{0}, R_{1}\right)\right)}\right.$ 
with some $R_{1}$ sufficienty small.

REMARK. With some modification the same method can be used to prove the analogous theorems for any bounded domain with sufficiently smooth boundary.

\section{REFERENCES}

1. M. Giaquinta and J. Nečas, On the regularity of weak solutions to nonlinear elliptic systems via Liouville's type property, CMUC 20, 1 (1979), 111-121.

2. - On the regularity of weak solutions to nonlinear elliptic systems of partial differential equations, J. reine angew. Math., 316 (1980), 140-159.

3. J. Nečas, On the regularity of weak solutions to nonlinear elliptic systems of partial differential equations, to appear in Editrice Tecnica Scientifiea, Pisa.

4. - A Necessary and sufficient condition for the regularity of weak solutions to nonlinear elliptic systems of partial differential equations, to appear in Abhandlungen Akad. der Wissensehaften der DDR.

Received November 17, 1980.

Charles University

1, Malostranske n. 25

11800 Praha 1, Czechoslovakia 



\section{PACIFIC JOURNAL OF MATHEMATICS}

\section{EDITORS}

DONALD BABBITT (Managing Editor)

University of California

Los Angeles, California 90024

\section{Hugo Rossi}

University of Utah

Salt Lake City, UT 84112

C. C. MOore and Arthur AGuS

University of California

Berkeley, CA 94720
J. DugundJI

Department of Mathematics University of Southern California Los Angeles, California 90007

R. FinN and J. Milgram Stanford University Stanford, California 94305

ASSOCIATE EDITORS
R. ARENS
E. F. BECKENBACH
B. H. NeumanN
F. WOLF
K. YOSHIDA

\section{SUPPORTING INSTITUTIONS}

UNIVERSITY OF ARIZONA

UNIVERSITY OF BRITISH COLUMBIA

CALIFORNIA INSTITUTE OF TECHNOLOGY

UNIVERSITY OF CALIFORNIA

MONTANA STATE UNIVERSITY

UNIVERSITY OF NEVADA, RENO

NEW MEXICO STATE UNIVERSITY

OREGON STATE UNIVERSITY
UNIVERSITY OF OREGON

UNIVERSITY OF SOUTHERN CALIFORNIA

STANFORD UNIVERSITY

UNIVERSITY OF HAWAII

UNIVERSITY OF TOKYO

UNIVERSITY OF UTAH

WASHINGTON STATE UNIVERSITY

UNIVERSITY OF WASHINGTON 


\section{Pacific Journal of Mathematics}

Vol. 99, No. $1 \quad$ May, 1982

Mariano Giaquinta, Jindrich Necas, O. John and J. Stará, On the

regularity up to the boundary for second order nonlinear elliptic systems . . 1

Siegfried Graf, Realizing automorphisms of quotients of product $\sigma$-fields . . 19

Alfred Washington Hales and Ernst Gabor Straus, Projective colorings . . . 31

Sandra Hayes, The weak Nullstellensatz for finite-dimensional complex

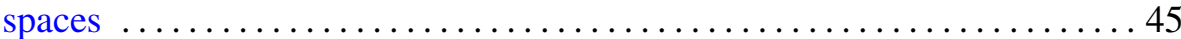

Gerald Norman Hile and Murray Harold Protter, The Cauchy problem

and asymptotic decay for solutions of differential inequalities in Hilbert

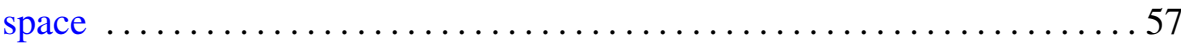

Robert D. Little, Projective space as a branched covering with orientable

branch set ......................................... 89

Jaroslav Mach, On the proximinality of Stone-Weierstrass subspaces . . . . . 997

John C. Morgan, II, On product bases ...................... 105

K. Balakrishna Reddy and P. V. Subrahmanyam, Altman's contractors

and fixed points of multivalued mappings . .................. 127

James Ted Rogers Jr., Decompositions of homogeneous continua . . . . . . . 137

Ahmed Ramzy Sourour, Characterization and order properties of

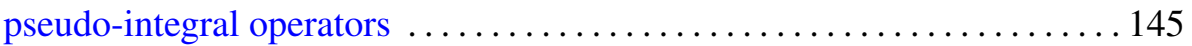

Robert Moffatt Stephenson Jr., Pseudocompact and Stone-Weierstrass

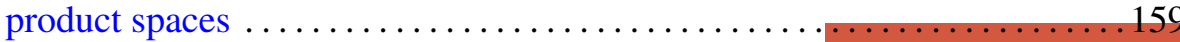

Bruce Stewart Trace, On attaching 3-handles to a 1-connected

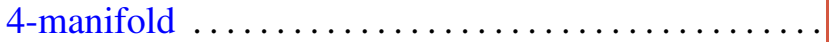

Akihito Uchiyama, The construction of certain BMO functions and the corona problem

Thomas Alva Whitehurst, An application of orthogonal polynomials to random walks ..............................

David J. Winter, Root locologies and idempotents of Lie and nonassociative algebras

William Robin Zame, The classification of uniform algebras on plane domains 